\title{
ADVANCED CLASSIFICATION OF OPTICAL AND SAR IMAGES FOR URBAN LAND COVER MAPPING
}

\author{
Damdinsuren Amarsaikhan \\ Institute of Geography and Geoecology, Mongolian Academy of Sciences, Mongolia - amarsaikhan@mas.ac.mn
}

Commission III, WG III/IV

KEY WORDS: Urban land cover, Optical, Synthetic aperture radar (SAR), Feature, Classification method

\begin{abstract}
:
The aim of this research is to classify urban land cover types using an advanced classification method. As the input bands to the classification, the features derived from Landsat 8 and Sentinel 1A SAR data sets are used. To extract the reliable urban land cover information from the optical and SAR features, a rule-based classification algorithm that uses spatial thresholds defined from the contextual knowledge is constructed. The result of the constructed method is compared with the results of a standard classification technique and it indicates a higher accuracy. Overall, the study demonstrates that the multisource data sets can considerably improve the classification of urban land cover types and the rule-based method is a powerful tool to produce a reliable land cover map.
\end{abstract}

\section{INTRODUCTION}

Remote sensing (RS) has provided an important source of information for urban land use and land cover classification, since the appearance of the first digital data sets (Stavrakoudis et al., 2011, Amarsaikhan et al., 2018). As it is one of the advanced techniques used to collect large amount of data with varying spatial resolutions without any physical contact with the object, the extracted information on land cover/use as well as individual objects is essential for rapid urban planning and management at all levels (Erener, 2013). However, urban areas are composed of many different types of objects, both natural and man-made which have different spectral characteristics in a RS image (Salah et al., 2019). For example, a simple building of building class appears as a complex structure with many architectural details surrounded by gardens, trees, grass, other buildings, roads, social and technical infrastructure and many other temporary objects (Pacifici et al., 2009). Therefore, urban area classification and object detection from RS images have been an important research topic for several decades.

Traditionally, multispectral RS data sets have been widely used for urban land-cover mapping (Amarsaikhan et al., 2012). However, due to the complex nature and diverse composition of the urban environment, the production of reliable and high quality urban land cover/use maps from the optical images is still a challenging task (Ban et al., 2010, Kadhim et al., 2016). In recent years, microwave images have been increasingly used for urban area classification (Attarchi, 2020). The studies have shown that SAR images may be the excellent basis for classifying, monitoring and analyzing urban conglomerations and their development over time especially, a large area mapping is under consideration (Dell'Acqua, 2009, Taubenböck et al., 2012, Amarsaikhan et al., 2018). As the multispectral and microwave images provide different information, their integration can be efficiently used for an improved urban mapping. It is clear that a combined use of the optical and SAR images will have a number of advantages because a specific urban feature, which is not seen on the passive sensor image may be observable on the microwave image and vice versa because of the complementary information provided by the two sources (Amarsaikhan et al., 2012, Amarsaikhan, Ganchuluun, 2015).
During the last decades, a significant progress has been made toward the development of new advanced active and passive RS sensors, with which accurate and detailed mapping of urban land cover and land use could become a reality (Hu, Wang, 2013, Zhu et al., 2018). However, as the urban areas are complex and diverse in nature and many features have similar spectral characteristics, it is still not easy to separate them by the use of ordinary feature combinations or by applying conventional techniques. Therefore, in urban area mapping, for differentiation of the spectrally similar or mixed classes, reliable features derived from multiple sources and an efficient classification technique should be used (Amarsaikhan et al., 2012). The aim of this study is to classify the features derived from optical and SAR data sets and produce a reliable urban land cover map using a rule-based classification method.

\section{TEST SITE AND DATA SOURCES}

As a test site, Ulaanbaatar, the capital city of Mongolia has been used. Ulaanbaatar city is situated in the central part of Mongolia, on the Tuul River, at an average height of $1350 \mathrm{~m}$ above sea level and currently has about 1.45 million inhabitants (MNSB, 2019). In the selected image frame of the city, it is possible to define such classes as builtup area, ger (Mongolian traditional dwelling) area, forest, grass, soil and water. The built-up area includes buildings of different sizes, while ger area includes mainly gers surrounded by fences. Figure 1 shows a Landsat 8 image of the test site, and some examples of its land cover.

In the current study, as data sources, Landsat 8 data of 15 July 2016 and Sentinel 1 SAR image of 06 July 2016 have been used. The Landsat 8 data has nine reflective bands (B1: $0.43-0.45 \mu \mathrm{m}$, B2: $0.45-0.51 \mu \mathrm{m}, \mathrm{B} 3: 0.53-0.59 \mu \mathrm{m}, \mathrm{B} 4: 0.64-0.67 \mu \mathrm{m}, \mathrm{B} 5$ : $0.85-0.88 \mu \mathrm{m}, \mathrm{B} 6: 1.57-1.65 \mu \mathrm{m}, \mathrm{B} 7: 2.11-2.29 \mu \mathrm{m}, \mathrm{B} 8: 0.50$ $0.61 \mu \mathrm{m}$ and $\mathrm{B} 9: 1.36-1.38 \mu \mathrm{m})$. The spatial resolution is $30 \mathrm{~m}$ for all bands, except band 8 which has a spatial resolution of $15 \mathrm{~m}$ (Munkhdulam et al., 2019). In the present study, channels $3,4,5,6,7$ have been used. Sentinel- 1 is the first of the Copernicus Programme satellite constellation conducted by the European Space Agency. This space mission is composed of two satellites, Sentinel-1A and Sentinel-1B that carry a C-band radar 
instrument. Based on a well-established heritage from the ERS, Envisat and Radarsat missions, Sentinel-1 carries a 12 m-long advanced SAR, working in C-band (Munkh-Erdene et al., 2018). In the present study, Sentinel $1 \mathrm{~A}$ VV polarization image with a spatial resolution of $10 \mathrm{~m}$ has been used.

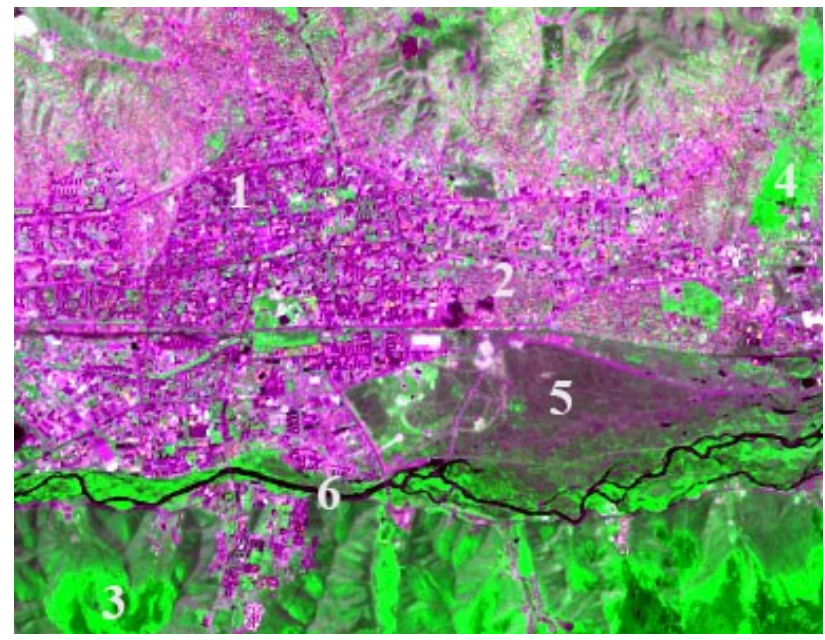

Figure 1. 2016 Landsat 8 image of the selected part of Ulaanbaatar city. 1-built-up area; 2-ger area; 3-forest; 4-grass; 5-soil; 6-water

\section{CO-REGISTRATION OF THE LANDSAT 8 AND SENTINEL-1A IMAGES}

Initially, a Landsat 8 image was georeferenced to a UTM map projection using 15 ground control points (GCP)s defined from a topographic map of the study area, scale 1:100,000. The GCPs have been selected on clearly delineated crossings of roads, streets and city building corners. For the transformation, a second-order transformation and nearest-neighbour resampling approach were applied and the related root mean square (RMS) error was 0.51 pixel. Then, the Sentinel 1A image was geometrically corrected and its coordinates were transformed to the coordinates of the georeferenced Landsat 8 image. In order to correct the Sentinel 1A image, 18 more regularly distributed GCPs were selected from different parts of the image. For the actual transformation, a second-order transformation was used. As a resampling technique, the nearest-neighbour resampling approach was applied and the related RMS error was 0.96 pixel. In both cases, each of the images was resampled to a pixel resolution of $20 \mathrm{~m}$.

\section{SPECKLE SUPPRESSION OF THE SENTINEL-1A IMAGE}

As microwave images have a granular appearance due to the speckle formed as a result of the coherent radiation used for radar systems; the reduction of the speckle is a very important step in further analysis. The analysis of the radar images must be based on the techniques that remove the speckle effects while considering the intrinsic texture of the image frame (Amarsaikhan et al., 2014). In the current study, four different speckle suppression techniques such as local region, kuan, frost and gammamap filters (ERDAS, 1999) of $3 \times 3$ and $5 \times 5$ sizes were compared in terms of delineation of urban features and texture information. After visual inspection of each image, it was found that the $3 \times 3$ gammamap filter created the best image in terms of delineation of different features as well as preserving content of texture information. In the output image, speckle noise was reduced with very low degradation of the textural information.

\section{FEATURE SELECTION AND A SUPERVISED CLASSIFICATION METHOD}

In any classification process, it is desirable to include different orthogonal features to increase its decision-making. In the present study, for this aim, the following feature combinations were determined:

1. All selected spectral bands of the Landsat 8 image.

2. Red, near infrared and second middle infrared (i.e., 4,5 and 7) bands of the Landsat data.

3. The combined features of Sentinel $1 \mathrm{~A}$ and Landsat images,

4. The principal component (PC) 1, PC2, PC3 and PC4 of the principal component analysis (PCA).

As seen, for the feature selection, in addition to the original data sets, the first four PCs obtained from the PCA have been chosen. The PCA is a data compression technique used to reduce the dimensionality of the multidimensional datasets. The bands of the PCA data are non-correlated and are often more interpretable than the source data. In $n$ dimensions, there are $n$ principal components. Each successive principal component is the widest transect of the ellipse that is orthogonal to the previous components in the $n$-dimensional space, and accounts for a decreasing amount of the variation in the data which is not already accounted for by previous principal components. Although there are $n$ output bands in a PCA, the first few bands account for a high proportion of the variance in the data (Amarsaikhan, Ganchuluun, 2015).

In the present study, the PCA has been performed using all available bands and the results are shown in table 1 . As can be seen from table $1, \mathrm{PC} 1$ is dominated by the variances of visible bands of the Landsat image and SAR band has insufficient influence on it. Although, it contained $58.86 \%$ of the overall variance, a visual inspection revealed that it contained less information related to the available urban classes. The Sentinel $1 \mathrm{~A}$ has a high loading in PC2. Here, all infrared bands of Landsat 8 have the negative loadings, however, visible bands have almost negligible effects. In PC3, near infrared band has a high loading and SAR band has the second highest loading. Although PC3 contained over $13 \%$ of the overall variance, visual inspection revealed that it contained some useful information related to the urban texture. PC4 is dominated by the negative and positive variances of the visible and infrared bands of the Landsat and it contained only $4.81 \%$ of the overall variance. Sometimes, useful information can be gathered from the bands with the least variances and these bands can show subtle details in the image that were obscured by higher contrast in the original image. However, the inspection of the PC5 and PC6 indicated that they mainly contained noise from the total data set. For the final analysis, the first four PCs that contained the largest portion of the overall variance have been selected. Figure 2 shows the comparison of the images obtained by the selected feature combinations.

\begin{tabular}{|c|cccccc|}
\hline & PC1 & PC2 & PC3 & PC4 & PC5 & PC6 \\
\hline Band 2 & 0.52 & 0.06 & -0.17 & -0.48 & -0.68 & 0.05 \\
Band 3 & 0.54 & 0.04 & -0.19 & -0.32 & 0.68 & -0.30 \\
Band 4 & -0.07 & -0.45 & 0.71 & -0.50 & 0.10 & 0.12 \\
Band 5 & 0.37 & -0.44 & 0.24 & 0.49 & -0.21 & -0.56 \\
Band 6 & 0.47 & -0.23 & -0.01 & 0.35 & 0.14 & 0.76 \\
SAR & 0.26 & 0.74 & 0.59 & 0.16 & -0.01 & -0.01 \\
Eigenvalue & 12541.1 & 4674.9 & 2931.2 & 1026.4 & 85.5 & 48.4 \\
Variance & 58.86 & 21.94 & 13.75 & 4.81 & 0.41 & 0.23 \\
$(\%)$ & & & & & & \\
\hline
\end{tabular}

Table 1. PC coefficients from Landsat 8 and Sentinel 1A images 
To classify the defined features, initially, the training samples had to be selected. To define the sites for the training signature selection from the combined optical and SAR images, several areas of interest (AOI) representing the available six classes (builtup area, ger area, forest, grass, soil and water) have been selected through accurate analysis. The separability of the training signatures was firstly checked in feature space and then evaluated using Jeffries-Matusita distance (Richards, Jia, 2006). The result of the separability measure is given in table 2 . The values of Jeffries-Matusita distance range from 0 to 2.0 and indicate how well the selected pairs are statistically separate. The values greater than 1.9 indicate that the pairs have good separability (ERDAS, 1999). After the investigation, the samples that demonstrated the greatest separability were chosen to form the final signatures. The final signatures included about 79-826 pixels.

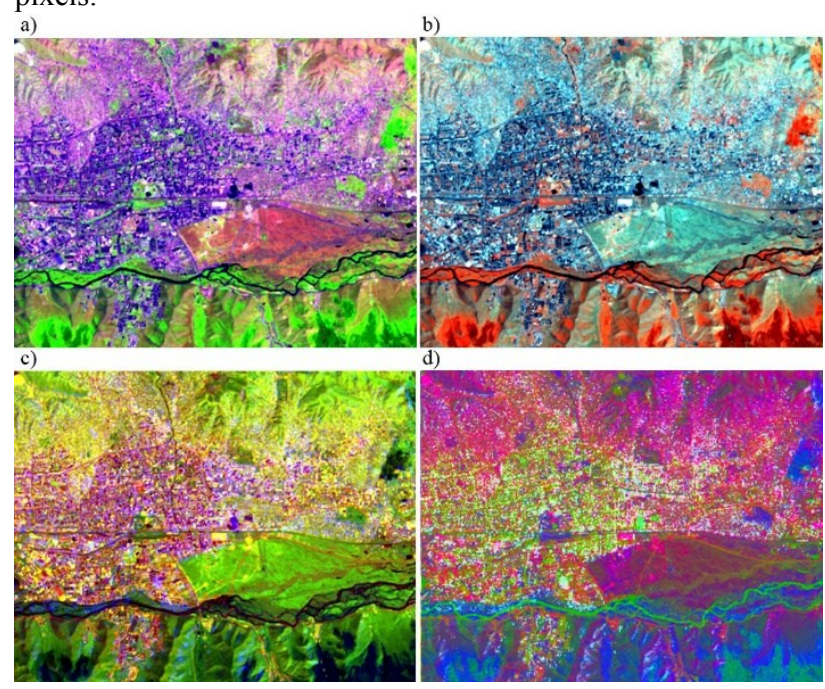

Figure 2. Comparison of the images obtained by the selected feature combinations: (a) the image obtained by Landsat band combinations ( $\mathrm{R}=\mathrm{B} 5, \mathrm{G}=\mathrm{B} 4, \mathrm{~B}=\mathrm{B} 3)$; (b) the image obtained by selected Landsat band combinations $(\mathrm{R}=\mathrm{B} 4, \mathrm{G}=\mathrm{B} 5, \mathrm{~B}=\mathrm{B} 6)$; (c) the image obtained by optical and SAR features $(R=B 3, G=B 5$, $\mathrm{B}=\mathrm{SAR})$; (d) $\mathrm{PC}$ image $(\mathrm{R}=\mathrm{PC} 1, \mathrm{G}=\mathrm{PC} 2, \mathrm{~B}=\mathrm{PC} 3)$

\begin{tabular}{|l|c|c|c|c|c|c|}
\hline & $\begin{array}{c}\text { Builtup } \\
\text { area }\end{array}$ & $\begin{array}{c}\text { Ger } \\
\text { area }\end{array}$ & Forest & Grass & Soil & Water \\
\hline $\begin{array}{l}\text { Builtup } \\
\text { area }\end{array}$ & 0.000 & 1.290 & 1.999 & 1.999 & 1.999 & 1.999 \\
\hline Ger area & 1.290 & 0.000 & 1.999 & 2.000 & 1.999 & 1.999 \\
\hline Forest & 1.999 & 1.999 & 0.000 & 1.726 & 2.000 & 1.999 \\
\hline Grass & 1.999 & 2.000 & 1.726 & 0.000 & 2.000 & 2.000 \\
\hline Soil & 1.999 & 1.999 & 2.000 & 2.000 & 0.000 & 1.999 \\
\hline Water & 1.999 & 1.999 & 1.999 & 2.000 & 2.000 & 0.000 \\
\hline
\end{tabular}

Table 2. The separabilities measured by Jeffries-Matusita distance

For the actual classification, a Mahalanobis distance and neural network classifiers have been used and compared. The Mahalanobis distance classifier is a parametric method, in which the criterion to determine the class membership of a pixel is the minimum Mahalanobis distance between the pixel and the class centre. The sample mean vectors and variance-covariance matrices for each class are estimated from the selected training signatures. Then, every pixel in the dataset is evaluated using the minimum Mahalanobis distance and the class label of the closest centroid is assigned to the pixel (Mather, Koh, 2011). Although, there are many neural network classification techniques, in most RS image analyses, a neural network method uses back- propagation for supervised learning, selecting a number of hidden layers. The users can choose different activation functions (Zayegh, Bassam, 2018). Learning occurs by adjusting the weights in the node to minimize the difference between the output node activation and the output. In the present study, a neural network classifier with 2 hidden layers has been applied. When the results from two classification methods compared, the performance of the neural network technique was worse than the performance of the Mahalanobis distance classifier. Therefore, for further comparison, the results of this method have been selected.

The classification results for the selected feature combinations obtained by the use of Mahalanobis method, are shown in figure $3(a-d)$. As seen from figure 3(a-d), the classification result of all bands of Landsat 8 gives the worst result, because there are high overlaps among two urban classes: builtup area and ger area. However, these overlaps decrease on the classified image of red and infrared bands. It can be explained by a fact that a fewer bands with statistically separable features can produce a better result than many bands with high overlaps. The combined use of optical and SAR data sets as well as the PC bands produced better results than the results of the Landsat 8 bands, but they still contain many mixed pixels for different classes. As seen, although multisource images give some improvement, it is still very difficult to obtain a reliable urban land cover map by the use of the standard technique, specifically on decision boundaries of the statistically overlapping classes.
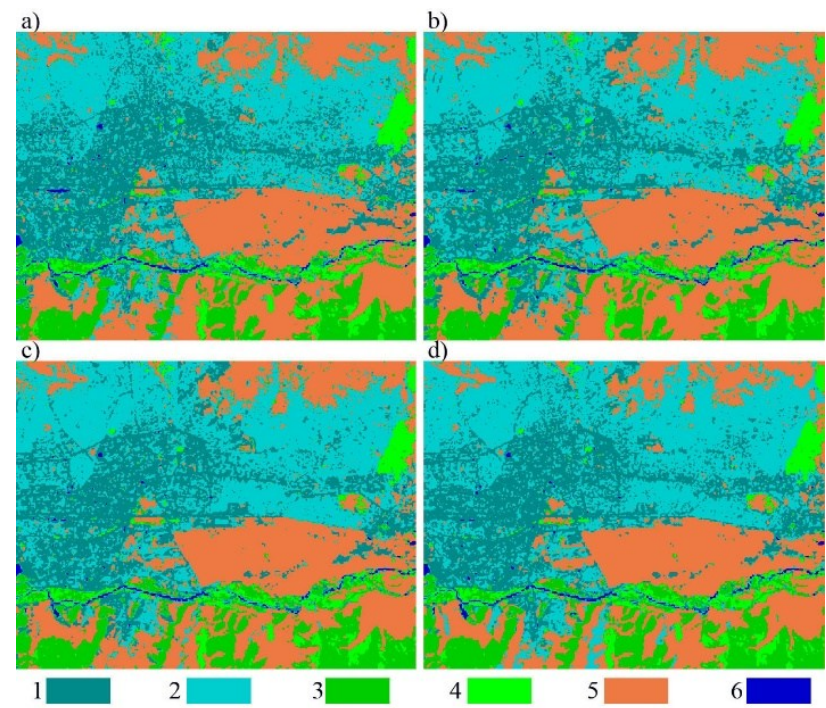

Figure 3. Comparison of the standard classification results for the selected classes (1-builtup area; 2-ger area; 3-forest; 4-grass; 5soil; 6-water). Classified images (a) using Landsat 8 bands, (b) using bands 4,5 and 6 of the Landsat 8 image, (c) using PCs, (d) using multisource bands

For the accuracy assessment of the classification results, different assessment methods such as overall accuracy, Kappa coefficient, producer's accuracy and user's accuracy are applied. As ground truth information, polygon-based pure pixels, ground truth images and randomly distributed points can be used. In the current study, we just wanted to compare the performances of the classification decision-rules. Therefore, for the accuracy assessment, because of its wide use, the overall accuracy method has been selected. This approach creates a confusion matrix in which reference pixels are compared with the classified pixels and as a result an accuracy report is generated indicating the percentages of the overall accuracy (Amarsaikhan et al., 2014). 
To select ground truth information, initially, the areas that contain pure pixels representing the selected classes were identified in different parts of the image. Then, based on a polygon-based approach, the AOIs containing 1268 purest pixels have been selected. The AOIs were selected on a principle that more pixels to be selected for the evaluation of the larger classes such as builtup area and soil than the smaller classes such as water. The overall classification accuracies for the selected classes are shown in table 3.

\begin{tabular}{|c|l|c|}
\hline No & \multicolumn{1}{|c|}{ The used band combinations } & $\begin{array}{c}\text { Overall accuracy } \\
(\%)\end{array}$ \\
\hline 1 & $\begin{array}{l}\text { All selected spectral bands of the } \\
\text { Landsat } 8\end{array}$ & 83.98 \\
\hline 2 & $\begin{array}{l}\text { Red, near infrared and second } \\
\text { middle infrared of the Landsat } 8\end{array}$ & 86.09 \\
\hline 3 & $\begin{array}{l}\text { Combined features of the } \\
\text { Sentinel 1A and Landsat } 8\end{array}$ & 87.89 \\
\hline 4 & PC 1,2,3 and 4 & 88.02 \\
\hline
\end{tabular}

Table 3. The overall classification accuracy of the classified images

\section{RULE-BASED CLASSIFICATION METHOD}

In general, it is very important to design an appropriate image processing procedure in order to successfully classify any digital data into a number of class labels. The effective use of different features derived from different sources and the selection of a reliable classification technique can be a key significance for the improvement of classification accuracy (Lu, Weng, 2007, Yu et al., 2016). In the present study, for the classification of urban land cover types, a rule-based algorithm has been constructed. As the reliable features, the first four PCs defined from the multisource images have been selected.

A rule-based approach is a part of knowledge-based techniques and it uses a hierarchy of rules or a decision tree describing the conditions under which a set of low-level primary objects becomes abstracted into a set of high-level object classes. The primary objects contain the user defined variables and include geographical objects represented in different structures, external programs, scalars and spatial models (Amarsaikhan et al., 2012).

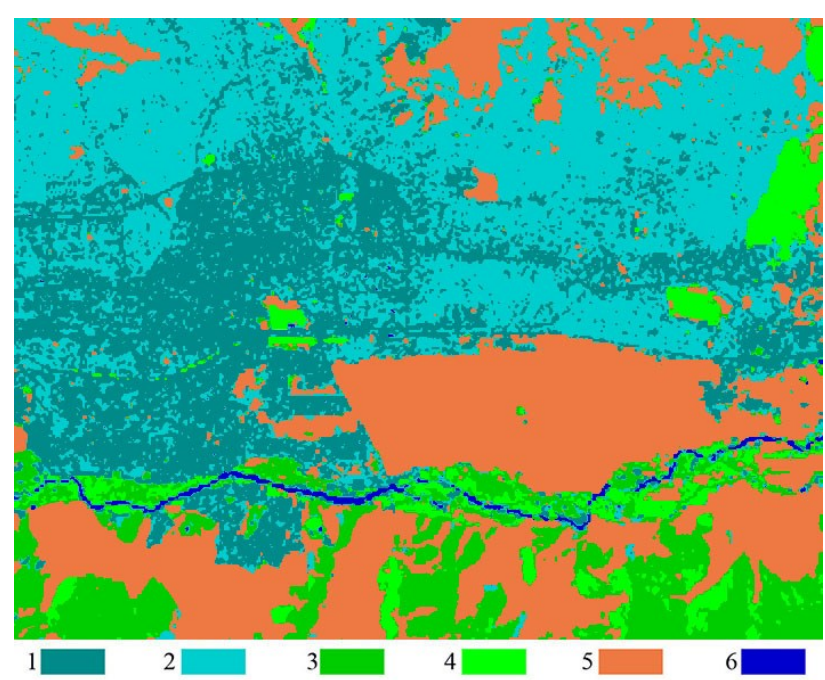

Figure 4. Classification result obtained by the rule-based classification.

(1-builtup area; 2-ger area; 3-forest; 4-grass; 5-soil; 6-water)
The constructed rule-based approach consists of a set of rules, which contains the initial image segmentation procedure based on a Mahalanobis distance rule and the constraints on spatial thresholds. It is clear that a spectral classifier will be ineffective if applied to the statistically overlapping classes such as builtup area and ger area, because they have very similar spectral characteristics in both optical and SAR images. For such spectrally mixed classes, classification accuracies can be improved if the spatial properties of the classes of objects could be incorporated into the classification process. These thresholds can be determined on the basis of different knowledge. In the current study, the spatial thresholds were defined on the basis of contextual knowledge about the test area. The contextual knowledge is based on the spectral and textural variations of the selected classes in different parts of the combined Landsat 8 and Sentinel 1A SAR images.

In the initial image classification, for separation of the statistically overlapping classes, only pixels falling inside of the spatial thresholds and the first four PCs of the PCA were used. The pixels falling outside of the spatial thresholds were temporarily identified as unknown classes and further classified using the rules in which other spatial thresholds were used. The final urban land cover map was created by combining all ancillary classification results. The image classified by this method is shown in figure 4 .

As seen from the classified image, the rule-based approach could very well separate the builtup area from the ger area compared to the results obtained by the traditional supervised method. The overall classification accuracy has been evaluated using the same set of regions containing the purest pixels as in the previously defined classifications and it demonstrated an improvement to $94.02 \%$.

\section{CONCLUSIONS}

The main aim of the research was to classify the multisource data sets using an advanced classification method and produce an improved urban land cover map. For the classification decision rule, four different feature combinations such as the original spectral bands of the Landsat 8 ; bands 4,5 and 7 of the Landsat 8; the combined bands of Sentinel 1A and Landsat 8 data sets; and first four PCs, were determined. To extract the reliable urban land cover information from the selected multispectral and SAR features, a rule-based classification algorithm that uses spatial thresholds defined from the contextual knowledge was constructed. The result of the rule-based method was compared with the results of the standard supervised classifications and it indicated a higher accuracy. Overall, the study demonstrated that the combined use of optical and microwave data sets could considerably improve the classification of urban land cover types and the rule-based method is a powerful tool to produce a reliable land cover map.

\section{REFERENCES}

Amarsaikhan, D., Saandar, M., Ganzorig, M., Blotevogel, H.H., Enkhjargal, D., 2012. Comparison of multisource image fusion methods and land cover classification. International Journal of Remote Sensing, 33(8), 2532-2550.

Amarsaikhan, D., Bolorchuluun, Ch., Ganchuluun, N., Ganzorig, M., Nergui, B., Enkhjargal, D., 2014. Fusion of optical and SAR images for the enhancement of urban features. CD-ROM Proceedings of the ACRS, Nay Pyi Taw, Myanmar. 
Amarsaikhan, D., Ganchuluun, N., 2015. Fusion and classification of multisource images for update of forest GIS. In: "Image fusion: principles, technology and applications". Nova Science Publishers, New York, USA, 83-121.

Amarsaikhan, D., Nyamjargal, E., Enkhjargal, D., MunkhErdene, A., Byambadolgor, B., Jargaldalai, E., Naranbat, S., Tsogzol, G., 2018. Urban land cover mapping in Mongolia using optical and LiDAR data. Proceedings of the ACRS, Kuala Lumpur, Malaysia.

Attarchi, S., 2020, Extracting impervious surfaces from full polarimetric SAR images in different urban areas. International Journal of Remote Sensing, https://doi.org/10.1080/01431161.2020.1723178.

Ban, Y., Hu, H., Rangel, I.M, 2010. Fusion of Quickbird MS and RADARSAT SAR data for urban land cover mapping: objectbased and knowledge-based approach. International Journal of Remote Sensing, 31(6), 1391-1410.

Dell'Acqua, F., 2009. The role of SAR sensors. In P. Gamba, \& M. Herold (Eds.), Global mapping of human settlements: Experiences, data sets, and prospects, 209-319.

ERDAS 1999, Field Guide, 5th edn (Atlanta, Georgia: ERDAS, Inc.).

Erener, E., 2013. Classification method, spectral diversity, band combination and accuracy assessment evaluation for urban feature detection. International Journal of Applied Earth Observation and Geoinformation, 21, 397-408.

Hu, S., Wang, L., 2013. Automated urban land-use classification with remote sensing. International Journal of Remote Sensing, 34(3), 90-803.

Kadhim, N., Mourshed, M., Bray, M., 2016. Advances in remote sensing applications for urban sustainability. Euro-Mediterr $J$ Environ Integr 1, 7, https://doi.org/10.1007/s41207-016-0007-4.

Lu, D., Weng, Q., 2007, A survey of image classification methods and techniques for improving classification performance. International Journal of Remote Sensing, 28,5, 823 -870 .

Mather, P.M., Koh, M., 2011. Computer Processing of RemotelySensed Images: an Introduction. Fourth edition (WileyBlackwell).

Mongolian Statistical Year Book (MNSB), 2019, National Statistical Office of Mongolia, Ulaanbaatar, Mongolia.

Munkhdulam, O., Atzberger, C., Chambers, J., Amarsaikhan, D., 2019, Mapping pasture biomass in Mongolia using Partial Least Squares, Random Forest regression and Landsat 8 imagery. International Journal of Remote Sensing, 40,8, 3204-3226.

Munkh-Erdene, A., Amarsaikhan, D., Ganzorig, M., Battsengel, V., Nyamjargal, E., Enkhjargal, D., Bolorchuluun, Ch., Byambadolgor, B., 2018. Applications of optical and radar images for forest resources study in Mongolia. Proceedings of the ACRS, Kuala Lumpur, Malaysia.

Pacifici, F., Chini, M., Emery. J., 2009. A neural network approach using multi-scale textural metrics from very high- resolution panchromatic imagery for urban land-use classification. Remote Sensing of Environment, 113, 1276-1292.

Richards, J.A., Jia, X., 2006. Remote Sensing Digital Image Analysis-An Introduction. Fourth Edition, Springer, ISBN: 3540-25128-6.

Salah, H.S., Ait-Aoudia, S., Rezgui, A., Goldin, S.E., 2019. Change detection in urban areas from remote sensing data: a multidimensional classification scheme. International Journal of Remote Sensing, 40(17), 6635-6679.

Stavrakoudis, D.G., Theocharis, J.B., Zalidis, G.C., 2011. A Boosted Genetic Fuzzy Classifier for land cover classification of remote sensing imagery. ISPRS Journal of Photogrammetry and Remote Sensing, 66, 529-544.

Taubenböck, H., Esch, T., Felbier, A., Wiesner, M., Roth, A., Dech, S., 2012. Monitoring urbanization in mega cities from space. Remote Sensing of Environment, 117, 162-176.

Yu, L., H. Fu, B. Wu, N. Clinton., Gong. P., 2016. Exploring the potential role of feature selection in global land-cover mapping. International Journal of Remote Sensing 37 (23), 5491-5504.

Zayegh, A. and Bassam, N.A., 2018, Neural network principles and applications, Digital Systems, IntechOpen, DOI: 10.5772/intechopen.80416.

Zhu, L., Suomalainen, J., Liu, J., Hyyppä, J., Kaartinen, H., Haggren, H., 2018. A review: remote sensing sensors. In: Rustamov, R.B., Hasanova, S., Zeynalova, M.H. (eds.) Multipurposeful Application of Geospatial Data, IntechOpen, London. 